\title{
Use of Social Media for Cancer Prevention Through Neighborhood Social Cohesion: Protocol for a Feasibility Study
}

Ingrid Oakley-Girvan ${ }^{1,2}$, MPH, PhD; Jessica L Watterson ${ }^{3,4}$, BA, MPH, PhD; Cheryl Jones ${ }^{5}$, PhD; Lauren C Houghton ${ }^{6,7}$, MSc, PhD; Marley P Gibbons ${ }^{6}$, BA; Kajal Gokal ${ }^{8}, \mathrm{PhD}$; Kate Magsamen-Conrad ${ }^{9}, \mathrm{PhD}$

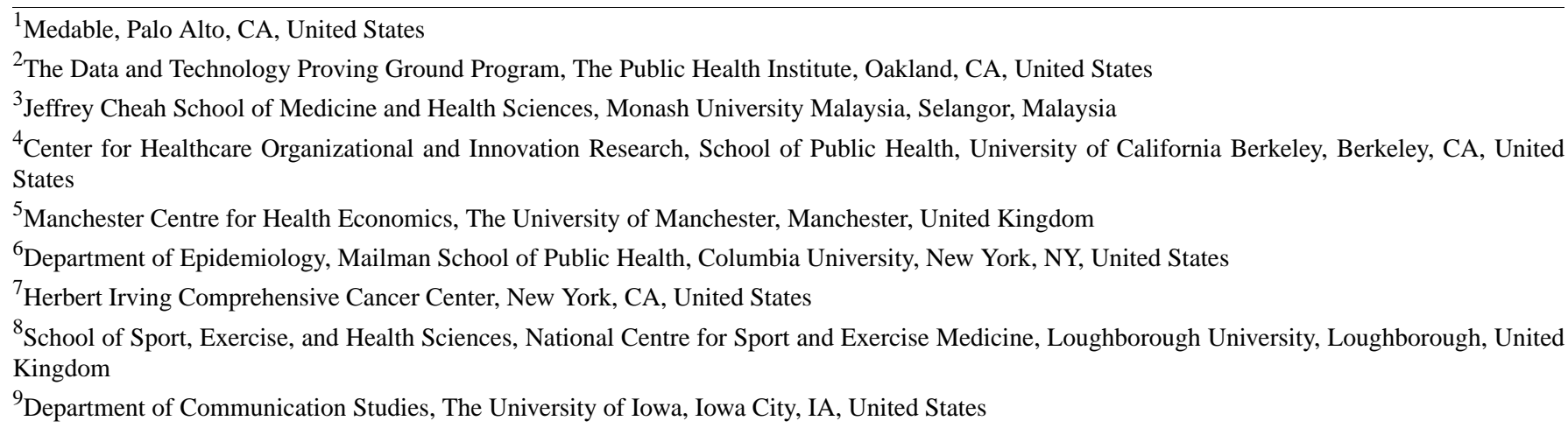

Corresponding Author:

Ingrid Oakley-Girvan, MPH, PhD

Medable

525 University Ave

Palo Alto, CA, 94301

United States

Phone: 14086562948

Email: ingrid@medable.com

\section{Abstract}

Background: Social cohesion is associated with healthier behaviors and better health outcomes, and therefore may offer a mechanism for promoting better health. Low socioeconomic status (SES) communities face higher rates of chronic disease due to both community- and individual-level factors.

Objective: The aim of this study is to leverage social cohesion to promote healthier behaviors and prevent chronic disease in a low SES community. This protocol outlines the methodology for a pilot study to assess the feasibility of an intervention (Free Time For Wellness [FT4W]) using a social networking platform (Nextdoor) with mothers living in an urban, low-income community to improve social cohesion and promote healthy behaviors.

Methods: The study will involve three phases: (I) co-designing the intervention with mothers in the neighborhoods of interest, (II) implementing the intervention with community leaders through the social networking platform, and (III) evaluating the intervention's feasibility. Phase I of the study will include qualitative data collection and analysis from in-depth, semistructured interviews and a co-design group session with mothers. Phases II and III of the study include a pre- and postintervention survey of participating mothers. Neighborhood-level data on social cohesion will also be collected to enable comparison of outcomes between neighborhoods with higher and lower baseline social cohesion.

Results: As of March 2021, recruitment and data collection for this study are complete. This protocol outlines our original study plan, although the final enrollment numbers and intervention implementation deviated from our initial planned methodology that is outlined in this protocol. These implementation learnings will be shared in subsequent publications of our study results.

Conclusions: Ultimately, this study aims to: (1) determine the barriers and facilitators to finding free time for wellness among a population of low-income mothers to inform the co-design process, and (2) implement and study the feasibility of an intervention that leverages social cohesion to promote physical activity in a community of low-income mothers. The results of this study will provide preliminary feasibility evidence to inform a larger effectiveness trial, and will further our understanding of how social cohesion might influence well-being.

International Registered Report Identifier (IRRID)： RR1-10.2196/28147 
(JMIR Res Protoc 2021;10(7):e28147) doi: 10.2196/28147

\section{KEYWORDS}

social cohesion; mothers; neighborhood; physical activity; social media; social; behavior; health outcomes; socioeconomic status; community health; chronic disease; social network; feasibility; wellbeing; cancer

\section{Introduction}

This protocol outlines the methodology for a pilot study to assess the feasibility of an intervention (Free Time For Wellness [FT4W]) using a social networking platform (Nextdoor) with mothers living in an urban, low-income community to improve social cohesion and to promote healthy behaviors.

Social cohesion describes the extent of connectedness and solidarity with groups [1], such as feelings of trust and inclusion in social settings [2]. Measures of social cohesion assess the degree to which individuals experience trusting relationships, cooperation, and participation in their communities [3]. High or average social cohesion is positively related to good self-rated health $[2,3]$. Social cohesion has also been found to be correlated with healthier behaviors and better health outcomes such as higher rates of physical activity [4]; lower rates of smoking, drinking, and depression [5]; lower BMI [6]; and lower rates of myocardial infarction [7]. Living in a cohesive community may improve health through the diffusion of health information and resources that enable individuals' engagement in healthy behaviors $[3,8]$. Social cohesion may also impact neighborhood safety [9] or social norms that reduce risky behavior and increase mutual respect, thereby reducing stress [3].

Given the linkages between social cohesion and health outcomes, our study aims to build social cohesion to promote healthy behavior using the social media app Nextdoor. As the prevalence of social media has risen in our society, its use has been studied for health issues such as tobacco use, diet, physical activity, and sexual practices [10]. A recent study of a social media-based pilot intervention for weight loss among adults with low socioeconomic status (SES) was found to be feasible, with results demonstrating high rates of engagement, increases in social support, and decreases in body weight among participants [11]. In addition, a recent meta-analysis of 22 studies of social media interventions for weight loss or related behaviors found a modest but statistically significant weight loss effect of 1 kilogram [12]. Facebook is the most commonly used tool for similar social media lifestyle intervention research [13]; therefore, this study will add to this body of literature by designing and evaluating an intervention using a newer social media tool (Nextdoor) that prioritizes connections between people in the same geographic area through address verification.

This study will focus on co-designing and implementing the intervention in the urban, low-income community of Washington Heights, New York City (NYC). Communities of low SES are at increased risk of developing conditions such as cancer, heart disease, diabetes, and other chronic diseases [3,14,15]. This increased risk is due to factors at many levels, including the environment, community, family, and individual [16]. At the community level, neighborhoods perceived as unsafe, hostile, isolating socially or culturally, or that are extremely polluted have been linked to poor self-rated health and higher mortality $[17,18]$. Greater levels of stress [19] and poorer sleep quality [20] occur among people who perceive their neighborhoods as unsafe and esthetically unpleasing. High levels of reported stress are also related to being less likely to engage in healthy behaviors [21], and the Centers for Disease Control and Prevention reported that individuals of low SES may also be more likely to smoke cigarettes, to be obese, to develop diabetes, and to experience preventable hospitalizations [22]. Therefore, this study will focus on a low SES community where the risk of chronic disease is higher and there is greater potential to make an impact on these health disparities.

Finally, this study will focus on co-designing and implementing the intervention with mothers, given their ability to influence the health behaviors of their households. According to Yuma-Guerrero and colleagues [23], social cohesion may improve mothers' engagement in physical activity, and thus directly impact individuals in that family because mothers make decisions that affect family health and model behavior for their children. Accumulating evidence [24] suggests that, ideally, cultivating risk-reduction behaviors begins in childhood, modeled by trusted caregivers and communities. We also selected mothers as the target population as they are more likely to have common interests and experiences that could facilitate building social cohesion, as opposed to a more heterogenous group. Therefore, this study will focus on cultivating social cohesion to promote healthy behaviors among mothers living in an urban, low-income community.

We hypothesize that an intervention using neighborhood-level social media to encourage behavioral activation and accountability to others will help to increase healthy behaviors among mothers living in an urban, low-income community.

\section{Methods}

\section{Purpose}

The purpose of this study is to co-design and assess the feasibility of the FT4W intervention. FT4W uses a social networking platform, Nextdoor, to improve social cohesion among mothers in a low-income, urban community (Washington Heights, NYC), with the ultimate goal of improving health behaviors that prevent chronic disease, such as physical activity. By focusing on building social cohesion, rather than explicitly discussing disease prevention or healthy behaviors, this intervention will employ a "stealth approach" to health promotion and will focus on addressing process motivation rather than on an explicit health outcome [25]. To our knowledge, no interventions using this approach to promote healthy behavior while building social cohesion have been performed in this target population with this social media platform. 
This pilot study has two aims. Aim 1 is to identify the barriers and facilitators to free time for wellness activities, and to co-design an intervention with mothers from an urban, low-income community. Aim 2 is to assess the feasibility of the intervention, leveraging social cohesion to promote healthy behaviors using Nextdoor among mothers in Washington Heights.

\section{Premise of the Study}

Although the exact intervention activities will be designed together with mothers from the target community, the research team has developed initial ideas for the intervention based on evidence, theory, and personal experience, and will seek feedback from mothers on these ideas. These initial ideas relate to the communication mode, program leadership, and intervention activities.

\section{Communication Mode: Nextdoor}

In our initial conceptualization, mothers would enroll in a Nextdoor group with others from their neighborhood. As outlined above, social media interventions show promise for improving social support and healthier behaviors, and Nextdoor was selected as the platform of interest by the research team because of its focus on physical proximity and safety through address verification. The relevant features of Nextdoor that will be considered for utilization in this intervention are: (i) connecting participants to others living in their neighborhood, (ii) creating a group of study participants where plans for group wellness activities in their neighborhood (and other topics of interest) can be discussed among participants, and (iii) group moderators can post information or reminders about upcoming events or activities. It is anticipated that the Nextdoor platform will be used for communication, whereas the wellness activities themselves will take place in person in the participants' neighborhood.

\section{Program Leadership: Community Champion}

We plan to engage a community champion to promote enrollment and participation in the intervention. We plan that their role will include sharing information about activities with the group, facilitating conversations among group members in the online platform, participating in activities, and generally promoting interest and engagement. The idea to utilize a community champion to facilitate the intervention stems from Rogers' diffusion of innovation theory, and subsequent evidence that suggests champions may be able to best influence others to enroll and maintain engagement in the program [26,27]. We plan to identify interested community champions during the activities of Aim 1 (interviews and workshop) and to offer a stipend as a gesture of appreciation for their time.

\section{Intervention Activities: Alternating Childcare and Exercise}

A potential intervention activity could include taking turns to watch children in a small group while other mothers go for a group walk, and then alternating roles so that all mothers have the opportunity to be active. This idea was inspired by personal experiences of the researchers, who had used Nextdoor in their own neighborhoods to arrange their own childcare and fitness activities.

Although these ideas are included here to illustrate the potential of this intervention, obtaining input from mothers during the co-design workshop is essential to ensure that the intervention fits their needs, and that they will be interested and motivated to participate.

We hypothesize that the co-design FT4W intervention can lead to behavior change through the framework outlined in Figure 1. Specifically, we hypothesize that the co-designed FT4W intervention will promote social cohesion, both at a community and individual level. A community champion will be used to facilitate the Nextdoor group intervention and to reinforce positive group dynamics. We expect that increases in social cohesion will result in greater community-level trust and accountability, as well as individual-level resources and activation (or cues to action). In turn, mothers will adopt healthier behaviors such as higher levels of physical activity, and will experience better health and greater capability. 
Figure 1. Hypothesized framework of behavior change. FT4W: Free Time For Wellness.

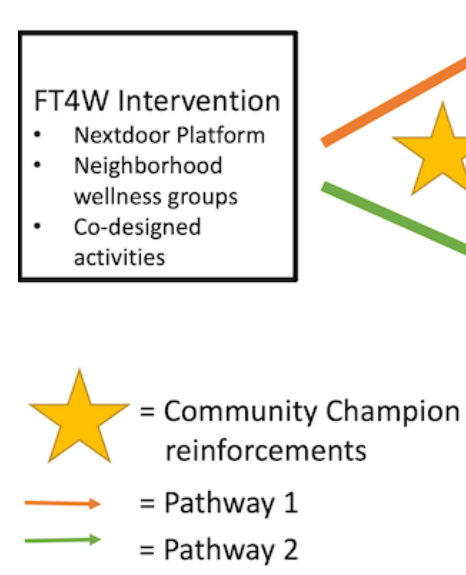

\section{Approach}

\section{Aim 1}

To achieve Aim 1, the researchers will perform qualitative interviews and a co-design workshop with mothers from the low-income, urban community of Washington Heights, NYC.

First, semistructured, open-ended interviews will be performed with mothers to understand the barriers and facilitators to free time for wellness. These interviews will also explore mothers' ability and willingness to use the social media platform Nextdoor. Approximately 12 mothers will be recruited for interviews from the Washington Heights community, although the final number will depend on when data saturation is met. Data saturation will be defined as "the degree to which new data repeat what was expressed in the previous data" [28]. In practice, this means that when the interviewers begin to hear the same comments being repeated in interviews, we will stop collecting new data and will begin analysis [28]. Recruiting will be performed through community groups, newsletters, Facebook groups, and listservs for mothers in the Washington Heights neighborhood, resulting in a convenience sample. The interviews will be recorded and transcribed, and the data will be inductively coded following grounded theory [29].

Second, a co-design workshop will be held with mothers in Washington Heights to design the content of the intervention. The workshop will include rotating, small group discussions at four "stations" to elicit ideas and feedback from mothers on four topics: (i) use of the Nextdoor platform and other communication technologies, (ii) ways to create additional free time in their schedules for wellness activities, (iii) the types of wellness activities they would like to participate in, and (iv) the characteristics they would like the community leader/facilitator to have. These topics were prioritized by the researchers as the main areas where feedback and input from participants were needed. The format of rotating small group discussions was chosen to facilitate easier conversation, where all participants would have the chance to speak and to give input on multiple topics.

A total of 20 mothers will be recruited to participate in the co-design workshop, starting with extending invitations to the mothers who participated in the interviews and asking them to invite other mothers from their community as well. In addition to this snowball sampling, flyers will be posted in the neighborhood to recruit additional mothers. Mothers' input will be audio-recorded and collected through notes taken by the research team during the workshop. Analysis of these qualitative data will be performed immediately following the workshop by reviewing the audio recordings and notes as a research team, discussing common themes emerging from the results, and deciding on the final components of the intervention through consensus. Using the design input from the mothers at the co-design session, the research team will decide on: (i) what the intervention wellness activities will be, (ii) how intervention group communication will take place, (iii) who will facilitate group communication and wellness activities, (iv) where the wellness activities will take place, and (v) when the wellness activities will take place (frequency).

\section{$\operatorname{Aim} 2$}

To achieve Aim 2, the research team will aim to enroll 30 mothers in the intervention group and will administer a baseline survey before the intervention begins and a follow-up survey after the intervention ends ( 4 months later). Mothers who attend the co-design workshop will be asked if they would like to participate in the intervention, and snowball sampling and neighborhood flyers will be used to recruit additional mothers until the target number is reached.

The baseline and follow-up survey will measure: physical activity (using the short version of the International Physical Activity Questionnaire [30]), individual-level perceptions of 
neighborhood social cohesion (using 4 questions from the National Health Interview Survey [31]), health status (using the EuroQol Five Dimension Five Level [32]), and capability (using the ICEpop CAPability measure for Adults [33]). The follow-up survey will also measure acceptability of the FT4W intervention (using questions to measure mothers' perceptions on whether the intervention is effective at freeing up their time to participate in wellness activities), perceptions of community among FT4W participants (through the Sense of Community Index [34]), and self-reported attendance to activities. In addition to the survey, data will also be collected on study retention (through attendance to intervention activities and participation in group communications), community-level interactions on Nextdoor (number of posts and replies among all Nextdoor members in Washington Heights neighborhoods during the study period), and the cost of the intervention. The relationships of these measured variables to our hypothesized framework of behavior change are outlined in Figure 2, showing how each variable will be measured through the Aim 2 data collection.

Figure 2. Hypothesized framework of behavior change with details on how each variable will be measured in Aim 2. NHIS: National Health Interview Survey; FT4W: Free Time For Wellness.

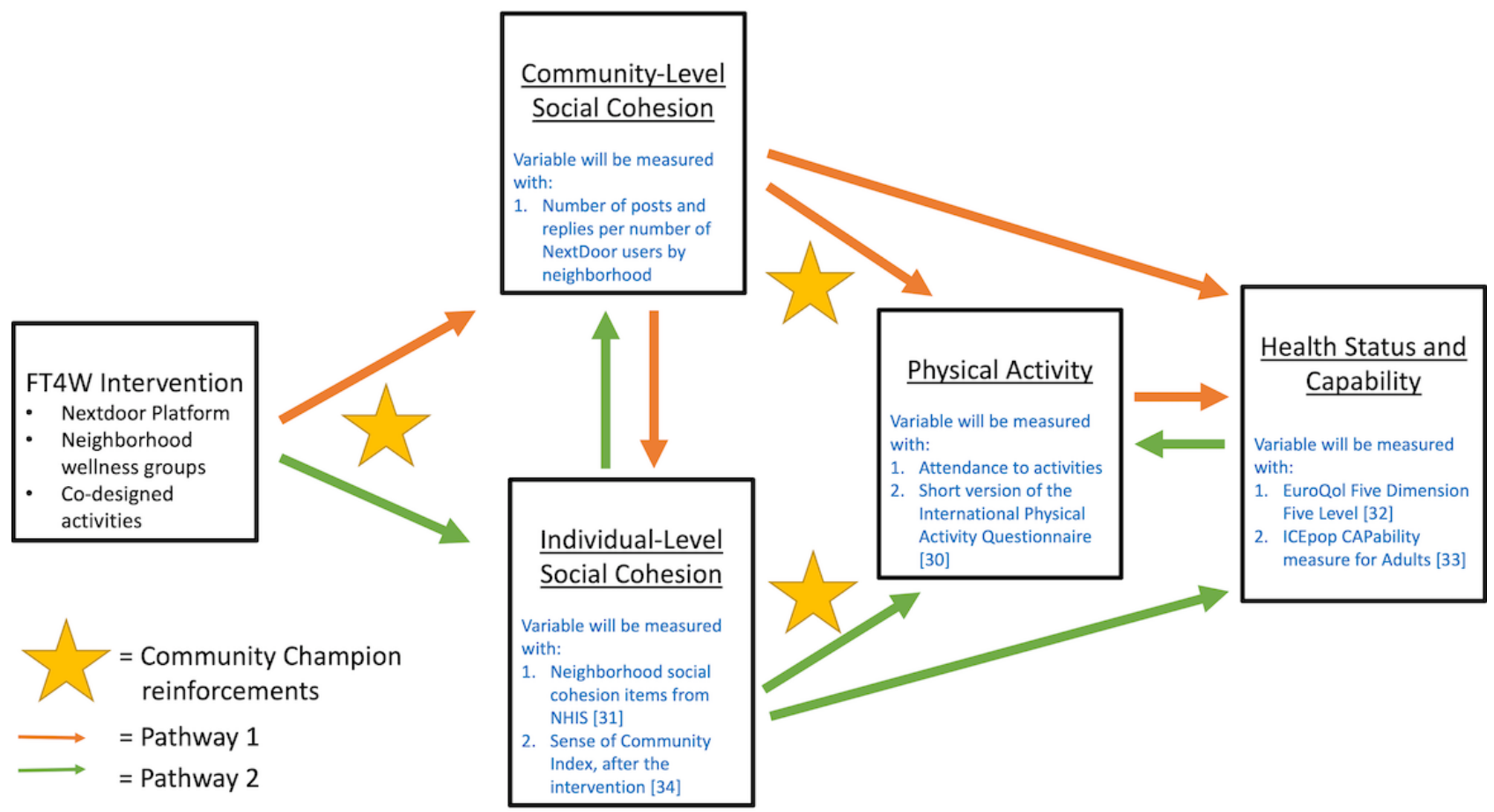

A descriptive analysis will be performed for each quantitative outcome measure described above. Given the small sample size, it will not be possible to make statistical inferences of the effectiveness of the intervention; however, the instruments used to collect the data will be assessed for their sensitivity and relevance for use in a future larger-scale trial. We will also perform an economic cost-benefit analysis because the introduction of a new intervention requires efficient resource allocation to achieve the greatest outcome. Economic evaluation is a tool that is used to compare the costs and benefits of two or more interventions [35]. To fully assess the cost and benefits of a new intervention, a trial with a sufficiently large sample size is required. However, it is important to consider the design of a cost-benefit (effectiveness) analysis from the inception of a new intervention. The purpose of the economic analysis for this study will be to test data collection methods (eg, baseline and follow-up surveys) to ascertain the most relevant and sensitive outcome measures that can be used to capture changes. Cost data will be estimated by collecting costs for services that would need to be funded for the intervention to succeed/function, including the cost of renting a community space for an intervention activity.

\section{Results}

As of March 2021, recruitment and data collection for this study is complete. This protocol outlines our original study plan, although final enrollment numbers and intervention implementation deviated from our initial planned methodology that is outlined in this protocol. These implementation learnings will be shared in subsequent publications of our study results.

\section{Discussion}

This protocol outlines the study methodology for a multilevel participatory community intervention to promote healthier behaviors among mothers in a low-income neighborhood. Unhealthy lifestyles and low rates of physical activity are potentially modifiable risk factors for many chronic diseases. Low SES communities are at especially elevated risk for developing chronic diseases, and they experience many barriers to better health. Low-cost, scalable programs that could be implemented regardless of geography, but tailored to the needs of neighborhoods, could result in a significant positive impact on national and potentially international health outcomes. 
This study involves a multidisciplinary research team with expertise in behavioral science and interventions, implementation science, health education, digital health, epidemiology, anthropology, psychology, health economics, and health communication. This study will also employ a multilevel approach to address barriers to healthy behavior at both the community and individual levels. Further, this study employs co-design methodology to engage the target community in the planning of the intervention, to ensure it meets their needs and is desirable and engaging. Finally, we have cultivated an informal partnership with the social media platform Nextdoor.
Similarly, we plan to engage with community services in Washington Heights to ensure that the intervention is local and accessible for mothers in the community.

The proposed multilevel mixed methods study will harness neighborhood-based social networking to improve social cohesion and ultimately chronic disease prevention through enhanced healthy behaviors among mothers in Washington Heights. If successful, this work could help reduce persistent disparities in chronic disease incidence and outcomes among communities with low SES.

\section{Acknowledgments}

A portion of this research was funded by Cancer Research UK (CRUK) through award reference C64528/A28666. CRUK provided feedback on the study design during the grant application process, but otherwise will play no role in the data collection, analysis, and interpretation of data, nor in writing of this manuscript or other manuscripts related to this study.

\section{Authors' Contributions}

All authors developed this study's aims and methodology. KC performed the background literature review. IG and KC drafted the manuscript. All authors provided feedback. JW added literature to the background section and revised the manuscript. All authors approved the final manuscript.

\section{Conflicts of Interest}

LCH is married to the Executive Director of one of the community service organizations where the intervention took place. Participants of the intervention received services that any member of New York City is eligible to receive. No money was exchanged. The remaining authors declare no conflict of interest.

\section{References}

1. Kawachi I, Berkman L. Social capital, social cohesion, and health. In: Berkman L, Kawachi I, Glymour M, editors. Social epidemiology. Oxford: Oxford University Press; Jul 2014.

2. Ou JY, Peters JL, Levy JI, Bongiovanni R, Rossini A, Scammell MK. Self-rated health and its association with perceived environmental hazards, the social environment, and cultural stressors in an environmental justice population. BMC Public Health 2018 Aug 03;18(1):970 [FREE Full text] [doi: 10.1186/s12889-018-5797-7] [Medline: 30075713]

3. Chuang Y, Chuang K, Yang T. Social cohesion matters in health. Int J Equity Health 2013 Oct 28;12(1):87 [FREE Full text] [doi: 10.1186/1475-9276-12-87] [Medline: 24165541]

4. Quinn T, Wu F, Mody D, Bushover B, Mendez DD, Schiff M, et al. Associations between neighborhood social cohesion and physical activity in the United States, National Health Interview Survey, 2017. Prev Chronic Dis 2019 Dec 19;16:E163 [FREE Full text] [doi: 10.5888/pcd16.190085] [Medline: $\underline{31858956}$ ]

5. Echeverría S, Diez-Roux AV, Shea S, Borrell LN, Jackson S. Associations of neighborhood problems and neighborhood social cohesion with mental health and health behaviors: the Multi-Ethnic Study of Atherosclerosis. Health Place 2008 Dec;14(4):853-865. [doi: 10.1016/j.healthplace.2008.01.004] [Medline: 18328772]

6. Cuevas AG, Kawachi I, Ortiz K, Pena M, Reitzel LR, McNeill LH. Greater social cohesion is associated with lower body mass index among African American adults. Prev Med Rep 2020 Jun;18:101098 [FREE Full text] [doi: 10.1016/j.pmedr.2020.101098] [Medline: 32382494]

7. Kim ES, Hawes AM, Smith J. Perceived neighbourhood social cohesion and myocardial infarction. J Epidemiol Community Health 2014 Nov 18;68(11):1020-1026 [FREE Full text] [doi: 10.1136/jech-2014-204009] [Medline: 25135074]

8. Dean L, Subramanian S, Williams DR, Armstrong K, Charles CZ, Kawachi I. The role of social capital in African-American women's use of mammography. Soc Sci Med 2014 Mar;104:148-156 [FREE Full text] [doi: 10.1016/j.socscimed.2013.11.057] [Medline: 24581073]

9. De Jesus M, Puleo E, Shelton RC, Emmons KM. Associations between perceived social environment and neighborhood safety: Health implications. Health Place 2010 Sep;16(5):1007-1013 [FREE Full text] [doi: 10.1016/j.healthplace.2010.06.005] [Medline: 20598624]

10. Cavallo DN, Chou WS, McQueen A, Ramirez A, Riley WT. Cancer prevention and control interventions using social media: user-generated approaches. Cancer Epidemiol Biomarkers Prev 2014 Sep;23(9):1953-1956 [FREE Full text] [doi: 10.1158/1055-9965.EPI-14-0593] [Medline: 25103820]

11. Cavallo D, Martinez R, Webb Hooper M, Flocke S. Feasibility of a social media-based weight loss intervention designed for low-SES adults. Transl Behav Med 2021 Apr 26;11(4):981-992. [doi: 10.1093/tbm/ibaa070] [Medline: 32716040] 
12. An R, Ji M, Zhang S. Effectiveness of social media-based interventions on weight-related behaviors and body weight status: review and meta-analysis. Am J Health Behav 2017 Nov 01;41(6):670-682. [doi: 10.5993/AJHB.41.6.1] [Medline: 29025495]

13. Waring ME, Jake-Schoffman DE, Holovatska MM, Mejia C, Williams JC, Pagoto SL. Social media and obesity in adults: a review of recent research and future directions. Curr Diab Rep 2018 Apr 18;18(6):34. [doi: 10.1007/s11892-018-1001-9] [Medline: 29671135]

14. Oates G, Jackson B, Partridge E, Singh K, Fouad M, Bae S. Sociodemographic patterns of chronic disease: how the mid-South region compares to the rest of the country. Am J Prev Med 2017 Jan;52(1S1):S31-S39 [FREE Full text] [doi: 10.1016/j.amepre.2016.09.004] [Medline: 27989290]

15. Hurlbert J, Beggs J, Haines V. Social networks and social capital in extreme environments. In: Lin N, Cook K, Burt R, editors. Social capital: theory and research. New York: Aldine de Gruyter; Jan 2002:28.

16. Golden SD, Earp JAL. Health Educ Behav 2012 Jun 20;39(3):364-372. [doi: 10.1177/1090198111418634] [Medline: 22267868]

17. Ajrouch KJ, Reisine S, Lim S, Sohn W, Ismail A. Perceived everyday discrimination and psychological distress: does social support matter? Ethn Health 2010 Aug;15(4):417-434 [FREE Full text] [doi: 10.1080/13557858.2010.484050] [Medline: 20582775]

18. Wen M, Hawkley LC, Cacioppo JT. Objective and perceived neighborhood environment, individual SES and psychosocial factors, and self-rated health: an analysis of older adults in Cook County, Illinois. Soc Sci Med 2006 Nov;63(10):2575-2590. [doi: 10.1016/j.socscimed.2006.06.025] [Medline: 16905230]

19. Henderson H, Child S, Moore S, Moore JB, Kaczynski AT. The influence of neighborhood aesthetics, safety, and social cohesion on perceived stress in disadvantaged communities. Am J Community Psychol 2016 Sep 30;58(1-2):80-88. [doi: 10.1002/ajcp.12081] [Medline: 27573035]

20. Hale L, Hill TD, Friedman E, Nieto FJ, Galvao LW, Engelman CD, et al. Perceived neighborhood quality, sleep quality, and health status: evidence from the Survey of the Health of Wisconsin. Soc Sci Med 2013 Feb;79:16-22 [FREE Full text] [doi: 10.1016/j.socscimed.2012.07.021] [Medline: 22901794]

21. Mouchacca J, Abbott GR, Ball K. Associations between psychological stress, eating, physical activity, sedentary behaviours and body weight among women: a longitudinal study. BMC Public Health 2013 Sep 11;13:828 [FREE Full text] [doi: 10.1186/1471-2458-13-828] [Medline: 24020677]

22. Meyer PA, Yoon PW, Kaufmann RB, Centers for Disease Control and Prevention (CDC). Introduction: CDC Health Disparities and Inequalities Report - United States, 2013. MMWR Suppl 2013 Nov 22;62(3):3-5. [Medline: 24264483]

23. Yuma-Guerrero PJ, Cubbin C, von Sternberg K. Neighborhood social cohesion as a mediator of neighborhood conditions on mothers' engagement in physical activity: results from the Geographic Research on Wellbeing Study. Health Educ Behav 2017 Dec 31;44(6):845-856. [doi: 10.1177/1090198116687537] [Medline: 28142286]

24. Mistry KB, Minkovitz CS, Riley AW, Johnson SB, Grason HA, Dubay LC, et al. A new framework for childhood health promotion: the role of policies and programs in building capacity and foundations of early childhood health. Am J Public Health 2012 Sep;102(9):1688-1696. [doi: 10.2105/AJPH.2012.300687] [Medline: 22813416]

25. Hekler EB, Gardner CD, Robinson TN. Effects of a college course about food and society on students' eating behaviors. Am J Prev Med 2010 May;38(5):543-547 [FREE Full text] [doi: 10.1016/j.amepre.2010.01.026] [Medline: 20227847]

26. Dearing JW. Applying diffusion of innovation theory to intervention development. Res Soc Work Pract 2009 Sep 01;19(5):503-518 [FREE Full text] [doi: 10.1177/1049731509335569] [Medline: 20976022]

27. Rojas L, Bahamon M, Lebron C, Montero-Zamora P, Pardo M, Wakefield M, et al. A feasibility trial of an online-only, family-centered preventive intervention for Hispanics: e-Familias Unidas. J Prim Prev 2021 Apr;42(2):97-124. [doi: 10.1007/s10935-020-00620-1] [Medline: 33532952]

28. Saunders B, Sim J, Kingstone T, Baker S, Waterfield J, Bartlam B, et al. Saturation in qualitative research: exploring its conceptualization and operationalization. Qual Quant 2018;52(4):1893-1907 [FREE Full text] [doi: 10.1007/s11135-017-0574-8] [Medline: 29937585]

29. Chun Tie Y, Birks M, Francis K. Grounded theory research: A design framework for novice researchers. SAGE Open Med 2019 Jan 02;7:2050312118822927 [FREE Full text] [doi: 10.1177/2050312118822927] [Medline: 30637106]

30. Hagströmer M, Oja P, Sjöström M. The International Physical Activity Questionnaire (IPAQ): a study of concurrent and construct validity. Public Health Nutr 2006 Sep 02;9(6):755-762. [doi: 10.1079/phn2005898] [Medline: 16925881]

31. National Health Interview Survey (NHIS). Centers for Disease Control and Prevention. 2018. URL: https://www.cdc.gov/ nchs/nhis/1997-2018.htm [accessed 2021-01-15]

32. Herdman M, Gudex C, Lloyd A, Janssen M, Kind P, Parkin D, et al. Development and preliminary testing of the new five-level version of EQ-5D (EQ-5D-5L). Qual Life Res 2011 Dec 9;20(10):1727-1736 [FREE Full text] [doi: 10.1007/s11136-011-9903-x] [Medline: 21479777]

33. Al-Janabi H, Flynn TN, Coast J. Development of a self-report measure of capability wellbeing for adults: the ICECAP-A. Qual Life Res 2012 Feb 20;21(1):167-176 [FREE Full text] [doi: 10.1007/s11136-011-9927-2] [Medline: 21598064]

34. Peterson NA, Speer PW, McMillan DW. Validation of a brief sense of community scale: Confirmation of the principal theory of sense of community. J Community Psychol 2007 Jan;36(1):61-73. [doi: 10.1002/jcop.20217] 
35. Drummond M, Sculpher M, Claxton K, Stoddart G, Torrance G. Methods for the economic evaluation of health care programmes. Fourth edition. Oxford, UK: Oxford University Press; 2015:464.

\title{
Abbreviations
}

FT4W: Free Time for Wellness

NYC: New York City

SES: socioeconomic status

\author{
Edited by G Eysenbach; submitted 23.02.21; peer-reviewed by J Goldthorpe, XF Zhong; comments to author 06.04.21; revised version \\ received 11.05.21; accepted 09.06.21; published 30.07.21 \\ Please cite as: \\ Oakley-Girvan I, Watterson JL, Jones C, Houghton LC, Gibbons MP, Gokal K, Magsamen-Conrad K \\ Use of Social Media for Cancer Prevention Through Neighborhood Social Cohesion: Protocol for a Feasibility Study \\ JMIR Res Protoc 2021;10(7):e28147 \\ URL: https://www.researchprotocols.org/2021/7/e28147 \\ doi: $\underline{10.2196 / 28147}$ \\ PMID: 34328445
}

CIngrid Oakley-Girvan, Jessica L Watterson, Cheryl Jones, Lauren C Houghton, Marley P Gibbons, Kajal Gokal, Kate Magsamen-Conrad. Originally published in JMIR Research Protocols (https://www.researchprotocols.org), 30.07.2021. This is an open-access article distributed under the terms of the Creative Commons Attribution License (https://creativecommons.org/licenses/by/4.0/), which permits unrestricted use, distribution, and reproduction in any medium, provided the original work, first published in JMIR Research Protocols, is properly cited. The complete bibliographic information, a link to the original publication on https://www.researchprotocols.org, as well as this copyright and license information must be included. 